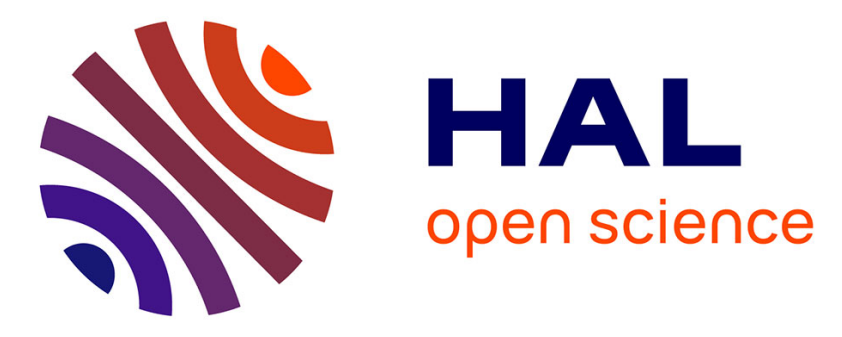

\title{
Shock-fitting and predictor-corrector explicit ALE Residual Distribution
}

Lorenzo Campoli, Pierrick Quemar, Aldo Bonfiglioli, Mario Ricchiuto

\section{To cite this version:}

Lorenzo Campoli, Pierrick Quemar, Aldo Bonfiglioli, Mario Ricchiuto. Shock-fitting and predictorcorrector explicit ALE Residual Distribution. Marcello Onofri; Renato Paciorri. Shock Fitting. Classical Techniques, Recent Developments, and Memoirs of Gino Moretti., Springer, 2017, Shock Wave and High Pressure Phenomena, 978-3-319-68427-7. hal-01625413

\section{HAL Id: hal-01625413 https://hal.science/hal-01625413}

Submitted on 27 Oct 2017

HAL is a multi-disciplinary open access archive for the deposit and dissemination of scientific research documents, whether they are published or not. The documents may come from teaching and research institutions in France or abroad, or from public or private research centers.
L'archive ouverte pluridisciplinaire HAL, est destinée au dépôt et à la diffusion de documents scientifiques de niveau recherche, publiés ou non, émanant des établissements d'enseignement et de recherche français ou étrangers, des laboratoires publics ou privés. 


\title{
Shock-fitting and predictor-corrector explicit ALE Residual Distribution
}

\author{
L. Campoli, P. Quemar, A. Bonfiglioli and M. Ricchiuto
}

\section{Introduction and main results}

This work is part of a long term effort to develop a modern unstructured shockfitting algorithm. The authors have so far shown the capabilities of their approach for steady flows in two $[12,13]$ and three [6] spatial dimensions, as well as some potential for time dependent moving shocks [7]. In all the mentioned works fitted shocks are treated as interior boundaries of zero thickness that are free to move throughout a triangular/tetrahedral mesh. This underlying unstructured triangulation/tetrahedrization covers the entire computational domain, and locally adapts to exactly fit the shock motion. The latter is ruled by the Rankine-Hugoniot jump relations which are in turn deduced from an approximate flow solution obtained by discretizing the compressible flow equations on either side of the discontinuity. To this end, in this work we will focus on the numerical solution of the perfect gas Euler equations. This contribution is a first step to evaluate the unsteady fitting method when schemes with different properties are used. We evaluate the results obtained with explicit residual distribution schemes in Arbitrary Lagrangian Eulerian (ALE) form, developed in [16, 5].

The paper investigates quantitatively and qualitatively the influence on the fitted solutions of the accuracy order (first or second here) of the method, as well as on

L. Campoli

Dipartimento di Meccanica e Aeronautica, Università di Roma -La Sapienza, Italy, e-mail: lorenzo.campoli@uniroma1.it

P. Quemar

LAGA, Université Paris 13 et LNHE, EDF, France e-mail: quemar@ math.univ-paris13.fr

A. Bonfiglioli

Scuola di Ingegneria, Università degli Studi della Basilicata, Potenza, Italy, e-mail: aldo.bonfiglioli@unibas.it

M. Ricchiuto

Team CARDAMOM, Inria Bordeaux Sud-Ouest, France, e-mail: mario.ricchiuto@inria.fr 


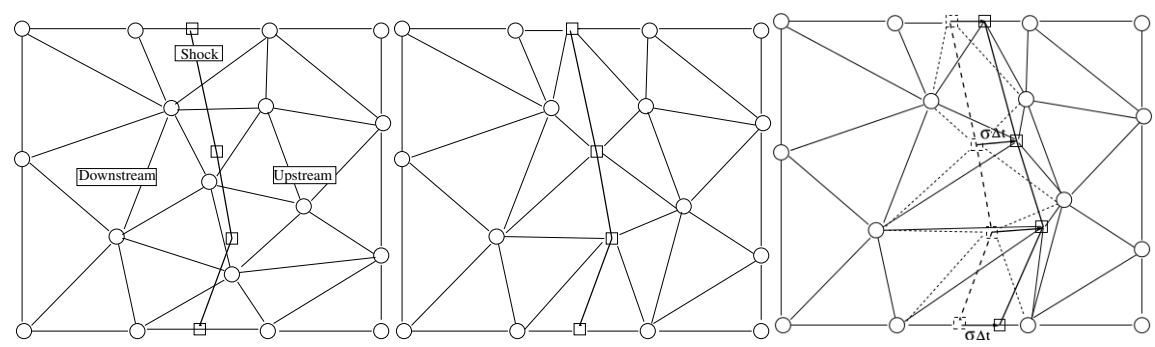

(a) Background mesh

(b) "Shock-fitted" mesh

(c) Shock-motion + deformation

Fig. 1: Shock-fitting: schematic illustration of some of the algorithmic ingredients.

the stabilization mechanism used. The main result is that accuracy seems to be the most influent factor.

\section{Numerical methods}

\subsection{Shock-fitting algorithm}

The unstructured shock-fitting algorithm consists of two key ingredients: 1) a local re-meshing technique that constructs a time-dependent mesh in which the fitted discontinuities are internal boundaries of zero thickness and 2) an algorithm for solving the Rankine-Hugoniot jump relations that provide the Lagrangian velocity of the discontinuity and an updated set of dependent variables within the downstream side of the fitted shock.

More precisely, in two space dimensions, the fitted shock fronts are made of polygonal curves, i.e., a connected series of line segments (which we call the shock edges) that join the shock points. These shocks are free to move throughout a background triangular mesh that covers the entire computational domain (see Fig. 1a). Starting from a background grid, at a given time level $n$ local mesh modifications are performed by re-generating a constrained Delaunay triangulation in the neighbourhood of the shock. This allows to ensure that the edges that make up the shock front are also part of the mesh that covers the entire computational domain (see Fig. 1b). The shock speed $\sigma_{s h}$ is computed from the Rankine-Hugoniot jump relations within each of the shock points. Using nodal values of shock speed and shock normal $\mathbf{n}$, the fitted shock front is moved in a Lagrangian manner, as shown in Fig. 1c. A second-order-accurate temporal integration of the shock trajectory is obtained using a predictor-corrector scheme [7].

In the smooth regions on either side of the shock, a vertex-centred ALE discretization, described in Section 2.2, allows to obtain an approximation of the flow variables on the mesh. The ALE formulation allows to embed naturally in the 
schemes the presence of the shock front as a moving boundary. This is schematically shown in Fig. 1c where dashed lines are used to show the edges of the triangular cells when the shock front is at time level $n$ and solid lines to show the same sides when the shock has reached time level $n+1$.

The interested reader can refer to [7] for additional details on the unsteady fitting procedure.

\subsection{Predictor-corrector ALE residual distribution}

We consider discretization methods for the Euler equations recast in the following ALE compact form:

$$
\partial_{t}(J \mathbf{w})+J \nabla \cdot(\mathrm{f}(\mathbf{w})-\sigma \mathbf{w})=0
$$

with $\mathbf{w}$ the array of conserved quantities (mass, momentum and energy), $f$ the conservative fluxes, $J$ the determinant of the Jacobian of the transformation between the reference and actual frame, and $\sigma$ the local deformation velocity. To discretize (1), we consider the two-step explicit Residual Distribution (RD) method developed in $[16,4,15]$, which can be written in the compact discrete form

$$
\left|C_{i}^{n+1}\right| \mathbf{w}_{i}^{n+1}=\left|C_{i}^{n+1}\right| \mathbf{w}_{i}^{*}-\Delta t \sum_{K \in \mathscr{D}_{i}} \Phi_{i}^{K}\left(\mathbf{w}_{h}^{n}, \mathbf{w}_{h}^{*}\right)
$$

where the fluctuations $\Phi_{i}^{K}$ define a splitting of the average element residual $\Phi^{K}$ defined by

$$
\sum_{j \in K} \Phi_{j}^{K}=\Phi^{K}=\frac{1}{\Delta t}\left(\int_{K^{n+1}} \mathbf{w}_{h}^{*}-\int_{K^{n}} \mathbf{w}_{h}^{n}\right)+\frac{1}{2} \Phi^{K}\left(\mathbf{w}_{h}^{n}\right)+\frac{1}{2} \Phi^{K}\left(\mathbf{w}_{h}^{*}\right)
$$

with

$$
\sum_{j \in K} \Phi_{j}^{K}=\Phi^{K}=\int_{\partial K^{n+1 / 2}}\left(\mathrm{f}\left(\mathbf{w}_{h}\right)-\sigma_{h} \mathbf{w}_{h}\right) \cdot n d s
$$

The vector $\sigma_{h}$ represents the mesh velocity with nodal values $\sigma_{i}=\left(x_{i}^{n+1}-x_{i}^{n}\right) / \Delta t$. The values $\mathbf{w}_{i}^{*}$ are obtained from a first order predictor which is computed as

$$
\left|C_{i}^{n+1}\right| \mathbf{w}_{i}^{*}=\left|C_{i}^{n+1}\right| \mathbf{w}_{i}^{n}-\Delta t \sum_{K \in \mathscr{D}_{i}} \tilde{\Phi}_{i}^{K}\left(\mathbf{w}_{h}^{n}\right)
$$

where now the fluctuations $\tilde{\Phi}_{i}^{K}$ are a splitting of the following geometrically nonconservative average steady cell residual [4]

$$
\sum_{j \in K} \tilde{\Phi}_{j}^{K}=\tilde{\Phi}^{K}=\int_{\partial K^{n+1 / 2}} \mathrm{f}\left(\mathbf{w}_{h}\right) \cdot n d s-\int_{K^{n+1 / 2}} \sigma_{h} \cdot \nabla \mathbf{w}_{h} d x
$$


Almost all the geometrical quantities in the scheme are evaluated on the half-time $n+1 / 2$ averaged configuration $\mathscr{T}_{h}^{n+1 / 2}$ which ensures

$$
\left|K^{n+1}\right|-\left|K^{n}\right|=\Delta t \int_{\partial K^{n+1 / 2}} \sigma_{h} \cdot n d s=0,
$$

thus the satisfaction of a Discrete Geometric Conservation Law (DGCL).

The properties of the method introduced above are determined by the definition of the split residuals $\Phi_{i}^{K}, \Phi_{i}^{K}$ and $\tilde{\Phi}_{i}^{K}$, which, following [16, 4, 15] have the form :

$$
\Phi_{i}^{K}=\sum_{j \in K} \frac{m_{i j}^{K^{n+1}} \mathbf{w}_{j}^{*}-m_{i j}^{K^{n}} \mathbf{w}_{j}^{n}}{\Delta t}+\frac{1}{2} \Phi_{i}^{K}\left(\mathbf{w}_{h}^{n}\right)+\frac{1}{2} \Phi_{i}^{K}\left(\mathbf{w}_{h}^{*}\right) \text { with } \Phi_{i}^{K}=\beta_{i} \Phi^{K}\left(\mathbf{w}_{h}^{n}\right)
$$

and similarly for $\tilde{\Phi}_{i}^{K}$. The $\beta_{j}$ are distribution matrices, uniformly bounded w.r.t the cell residuals (6), (3), while the $m_{i j}^{K}$ s are mass matrix entries consistent with the definition of the spatial distribution [16]. Independently of the specific construction of these quantities, the above definitions give a scheme which is formally second order accurate in space and time, fully conservative, and verifying the DGCL. The interested reader is referred to $[4,3,15,16]$ for more details. In practice, we have used two main classes of methods: multidimensional upwind (MU) schemes, and centered schemes with some form of stabilization. In particular, the computations shown in the paper will compare results using the first order linear and monotone $\mathrm{N}$ scheme and the second order linear LDA scheme. These are MU distribution methods. A nonlinear method obtained by blending these two, and referred to as the LDAN scheme, is also tested. Two non-upwind methods are also considered, in particular, the explicit predictor-corrector formulation of the second order linear Streamline-Upwind (SU) method proposed in [16] (see also [9] and references therein), and the nonlinear blended central (Bc) discretization obtained when blending the SU method with a limited Lax-Friedrich's distribution. The interested reader may consult the above mentioned references for more details.

\section{Numerical results}

\subsection{Shock-expansion interaction}

We start by investigating the behaviour of the schemes on the computation of an expansion fan interacting with a moving shock. The problem involves a rectangular cavity initially filled with an inviscid perfect gas at rest, as shown in the sketch of Fig. 2a. The left end of the domain acts as a piston which is impulsively set into motion, generating a planar shock wave moving towards the closed (right) end of the domain. Given the density ratio across the shock wave, all other kinematic and thermodynamic quantities follow from the Rankine-Hugoniot (R-H) jump relations 
and are summarized in Tab. 1. The upstream and downstream dimensionless quantities have been labelled with the subscripts 1 and 2 in Tab. 1. The Mach number in a reference frame attached to the shock is denoted by $M_{r}$, and its upstream value coincides with that of the dimensionless shock speed $W_{s}$, since $a_{1}$ has been chosen as the reference velocity.

After the impulsive startup, the piston starts slowing down at $t=t_{0}+t_{s}$; its velocity and position are prescribed analytically by:

$$
u_{p}(t)=\left\{\begin{array}{ll}
u_{2} & t_{0}<t \leq 0 \\
u_{2} \exp ^{-t} & t \geq 0
\end{array}, x_{p}(t)=\left\{\begin{array}{ll}
u_{2} t & t_{0}<t \leq 0 \\
u_{2}\left(1-\exp ^{-t}\right) & t \geq 0
\end{array},\right.\right.
$$

where $t_{0}=-t_{s}$ and:

$$
t_{s}=\frac{x_{s h}^{0}}{W_{s}-u_{2}} \simeq 0.3755
$$

\begin{tabular}{|c|c|c|c|c|c|c|}
\hline & $\rho$ & $u$ & $v$ & $p$ & $M_{r}$ & $a$ \\
\hline \hline Upstream (1) & 1.4 & 0.0 & 0.0 & 1.0 & 1.5336 & 1.0 \\
\hline Downstream (2) & 2.6872 & 0.7346 & 0.0 & 2.5772 & 0.6895 & 1.1588 \\
\hline
\end{tabular}

Table 1: Shock-expansion interaction: initial conditions.

The flow evolution is sketched in the $x-t$ plane of Fig. 2c. We start the simulations at $t=0$ with the shock located $x_{s h}^{0}=0.3$ unit lenghts ahead of the piston and we initialize the solution using the initial conditions listed in Tab. 1.

The tail of the expansion wave (marked by arrows in Fig. 2c) is the straight, characteristic line of slope $u_{2}+a_{2}$ that is shed from the origin of the space-time plane and divides the expansion wave from the uniform, shock-downstream region, labelled 2 in Fig. 2. Up to time:

$$
\hat{t}=\frac{x_{s h}^{0}}{\left(u_{2}+a_{2}\right)-W}=\frac{x_{s h}^{0}}{\left(1-M_{r, 2}\right) a_{2}} \simeq 0.8339
$$

when the tail of the expansion wave meets the shock trajectory, the expansion region is a simple wave, so that an analytical solution can be computed as described, for instance, in [20, $\S 4.1 .5]$. At later times, the expansion wave is reflected off the moving shock as an expansion wave of the opposite family, the shock starts reducing its speed and the region of the $x-t$ plane that is between the moving piston and the moving shock ceases to be a simple wave.

Simulations have been advanced until the final time $t_{2}=2.8$ when the shock is located at $x_{s h}^{t_{2}} \simeq 4.43$ and has almost reached the closed (right) end of the cylinder. We present results obtained on a background grid of 639 nodes, 12448 triangles, with a shock front discretized by 41 points (cfr. right picture of Fig. 2a). During the time evolution, the streamwise size of the computational domain is reduced because of the piston's motion. All nodes of the background mesh move as the time elapses, 


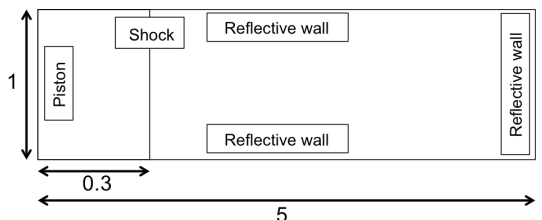

(a) Computational set-up at $t=0$.

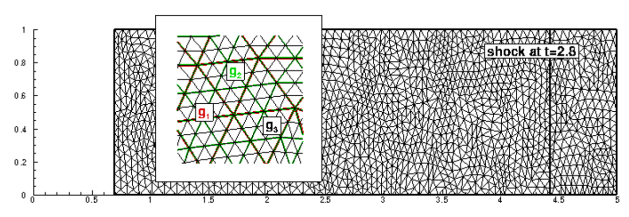

(b) Nested meshes and shock position at $t=2.8$.

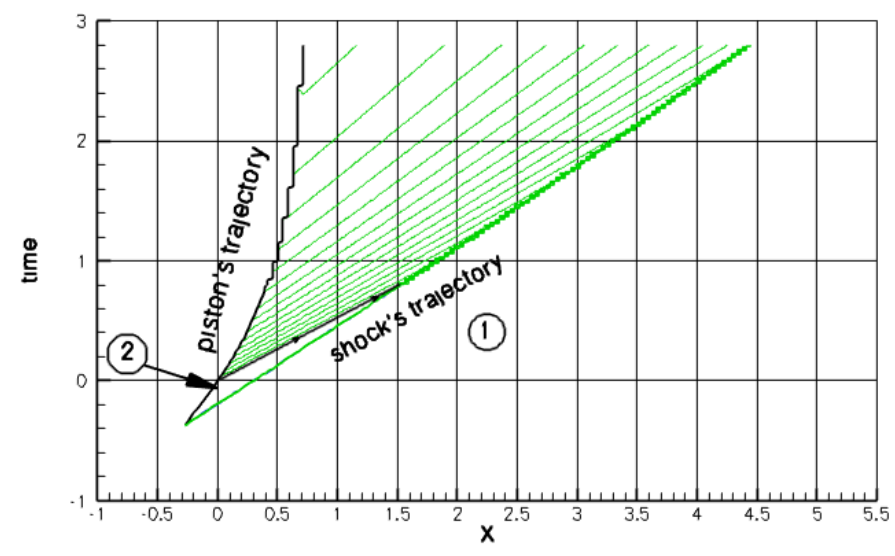

(c) Pressure iso-contours in the space-time plane.

Fig. 2: Shock-expansion interaction.

but the topology of the mesh does not change. At any time instant, the location of the nodes of the background mesh is prescribed using the following equation:

$$
x_{i}(t)=x_{i}(0)+x_{p}(t)\left(1-\frac{x_{i}(0)}{L}\right)
$$

where $L=5$ is the streamwise length of the computational domain at $t=0$.

Figure 3 provides the pressure field at three subsequent time instants for all the five different spatial discretization schemes and the two shock-modelling schemes. Apart from the excessive shock-thickness of the shock-capturing solutions, it is clear that shock-fitting provides a much cleaner representation of the shock-downstream region. Observe, in particular, that the crossflow non-uniformities are considerably less pronounced in the shock-fitting calculations.

Figure 4 shows the streamwise pressure profiles at two subsequent time instants for all the five spatial discretization schemes. Note that the figures report all the data points in the mesh. The superiority of the shock-fitting calculations is evident, particularly in the reduction of cross-shock spurious variations, despite the 
fact that both the fitted, and captured solutions are represented on unstructured triangulations with almost identical topologies. We do observe an impact of the numerical discretization chosen. The upwind or centered nature of the method does not seem to affect the results as much as the resolution of the scheme. The $\mathrm{N}$ scheme clearly provides the "wrong boundary conditions" for the shock, which seems slighly miscplaced w.r.t. the analytical solution, as one can see in the right frame of Fig. 4a. Shock amplitude and position are instead perfectly matched by the high order schemes, with perhaps a barely visible off-set present only in the LDAN result, shown in Fig. 4c. These differences are clearly much more important in the captured solutions: the linear, first-order-accurate $\mathrm{N}$ scheme smears the shock-profile over a considerable streamwise extent; as expected, the linear, secondorder-accurate, but non-monotone LDA and SU schemes give rise to overshoots around the discontinuity; the non-linear, second-order-accurate LDAN scheme is monotonicity preserving, but the otherwise similar $\mathrm{Bc}$ scheme also overshoots the shock profile.

\subsection{Shock-vortex interaction}

The interaction between a shock and a vortex has been frequently reported in the literature as a tool for understanding the mechanisms of noise generation due to the interaction between a shock-wave and a turbulent flow [14, 11, 10, 8]. This problem has also been frequently used as a code verification benchmark [23, 1, 18]. Shock-fitting calculations of shock-vortex interactions have been previously reported in $[14,21,2]$.

A uniform, supersonic stream, characterised by a shock-upstream Mach number $M_{s}=\left|\mathbf{u}_{\infty}\right| / a_{\infty}$, carries a vortex, from the left to the right of Fig. 5, towards a stationary normal shock. The computational domain, which is sketched in Fig. 5 along with the boundary conditions applied along its boundaries, is the rectangle $[0,2 L] \times[0, L]$. At the initial time, $t=0$, the vortex is centred in $\left(x_{v} / L, y_{v} / L\right)=(0.5,0.5)$ and the shock is located $0.2 L$ downstream of the vortex centre. The vortical structure we have used, which is an exact solution of the steady Euler equations, consists in a clockwise vortex characterised by a purely tangential velocity component. More precisely, using a polar coordinate system with the origin at the centre of the vortex and moving at constant speed $\left|\mathbf{u}_{\infty}\right|$, the perturbation velocity field $\tilde{\mathbf{u}}$ reads:

$$
\begin{aligned}
\tilde{u}_{\theta} & =-\varepsilon\left|\mathbf{u}_{\infty}\right| \tau e^{\alpha\left(1-\tau^{2}\right)} \\
\tilde{u}_{r} & =0
\end{aligned}
$$

In Eq. (10) $\tau=r / r_{c}$ is the non-dimensional radial distance from the pole of the moving reference frame and $\varepsilon, \alpha$ and $r_{c}$ are user-defined parameters that control 
the shape and magnitude of the perturbation. This particular solution of the Euler equations features a divergence-free velocity field, which implies that density is constant along the streamlines and a non zero vorticity field. Since the free-stream flow is isentropic, the thermodynamic variables can be easily obtained from the linear momentum equation, and Crocco's form of the steady momentum equation implies that there must be a gradient of the perturbation total enthalpy in the radial direction, so that total enthalpy also changes across the streamlines.

A vortex Mach number, $M_{v}$, can be defined as the ratio between the maximum velocity perturbation and the sound speed, $a_{\infty}$, of the shock-upstream flow: $M_{v}=\max \left(\tilde{u}_{\theta}\right) / a_{\infty}$. For the particular vortical structure used here, the vortex Mach number becomes:

$$
M_{v}=\varepsilon M_{s} \frac{e^{\left(\alpha-\frac{1}{2}\right)}}{\sqrt{2 \alpha}}
$$

Making the same choice of constants in Eqs. (10) and (11) also used in [23, 1, 18]: $\alpha=0.204$ and $r_{c} / L=0.05$, the vortex radius is about $0.35 L$, the maximum velocity perturbation occurs at about $0.11 L$ from the vortex centre and the strength $\varepsilon$ of the velocity perturbation follows from Eq. (11), once $M_{s}$ and $M_{v}$ have been set.

The topological pattern that arises once the vortex impinges on the shock depends upon both the shock and vortex strengths. We shall hereafter refer to the taxonomy adopted by Grasso and Pirozzoli [8], who define weak shock-vortex interactions as those that do not exhibit any shock reflection and strong shock-vortex interactions as those that feature secondary shocks; strong interactions can be further classified depending on whether a regular reflection or a Mach reflection occurs. The same authors identify in the $\left(M_{s}, M_{v}\right)$ pair the two independent variables that govern the interaction: when the vortex Mach number is sufficiently low, weak interactions always occur; however, at a given shock strength, an increase in the vortex Mach number above the $M_{v} \approx 0.1 \div 0.2$ threshold causes the shock to fold thus producing reflected and diffracted shocks that yield either a regular or a Mach reflection, depending upon the value of $M_{v}$.

In the present work, we have set the shock and vortex Mach numbers respectively equal to $M_{s}=2.0$ and $M_{v}=0.2$, so that a weak interaction occurs. The reason for doing so is that the present shock-fitting algorithm is currently unable to automatically identify and follow the appearance of those secondary shocks that arise when a strong interaction takes place. Nonetheless, as demonstrated in [7], strong interactions can be simulated with the available algorithm, thanks to a hybrid mode of operation whereby the normal shock is fitted and the secondary shocks are captured. This hybrid modelling is however not suited for the grid-convergence analysis which will be performed hereafter, since it is impossible to separately identify the effects of the two different shock-modelling practices. Therefore, only weak interactions will be considered here and simulations will be performed using each of the five schemes, both in shock-fitting and shock-capturing mode.

When simulations are run in shock-fitting mode, the linear LDA and SU schemes, that are not monotonicity preserving, can be used without giving rise to oscillations because they are used in smooth regions of the flowfield. The use of non-linear 
schemes is unnecessary in this case, but non-linear scheme have also been tested in conjunction with shock-fitting to allow a comparison with the shock-capturing calculation using the same scheme.

When simulations are run in shock-capturing mode, it should be expected and it is indeed observed that the linear schemes give rise to wiggles around the captured shock wave. Nonetheless, shock-capturing calculations with linear schemes have also been performed to allow a comparison with the shock-fitting calculation using the same scheme.

A qualitative view of the solutions obtained with the two approaches is given in Fig. 6. The pictures show the total enthalpy contours in the solutions obtained with the LDA and LDAN schemes with shock capturing and fitting on the finest mesh level of table 2. Note that the fitted results are virtually indistinguishable, and only those of the LDA are reported. As for the shock-expansion interaction, the qualitative difference is already quite striking from the comparison of the contours. In particular, besides the oscillations related to the approximation of the shock, we can see clearly that the contours downstream of the discontinuity are much less smooth in the captured solutions. This is more visible in the LDAN results (Figs. 6d6f), however a similar behaviour is also present in the LDA solutions. The fitted computations, on the other hand, show very nice and smooth contours.

In order to perform a quantitative comparison among the various combinations of spatial discretization schemes and shock-modelling practices, we have used the following global measure:

$$
S_{h}(t)=\frac{1}{|\Omega|} \int_{\Omega} s_{h}(x, t) d \Omega
$$

where $s=p \rho^{-\gamma}$ is entropy and we have computed the corresponding discretization error and order-of-convergence. To perform a grid convergence analysis, the computational domain has been discretized using a sequence of four nested Delaunay triangulations, hereafter labelled $g_{1}$ to $g_{4}$. The coarsest $g_{1}$ mesh has been created using the Triangle code [22] by specifying a maximum area constraint, whereas the other three grid levels have been obtained by recursive subdivision of each triangle of the coarser level into four sub-triangles. Table 2 summarizes the characteristics of the various meshes that have been used both in the shock-capturing computation and as background triangulations in the shock-fitting computation. The dimensionless, characteristic spatial size $h_{k}$ of grid $k$ has been set equal to the uniform spacing along the boundaries of the computational domain; observe that both $h_{k}$ and the dimensionless time-step length $\Delta t_{k}$ are halved when moving from grid level $k$ to the finer grid level $k+1$.

Whenever an exact, or manufactured solution to the set of governing PDEs is available, the discretization error can be evaluated straightforwardly. However, when the 


\begin{tabular}{|c|c|c|c|c|}
\hline grid & N. of triangles & N. of gridpoints & $\Delta t_{k}$ & $h_{k}$ \\
\hline \hline$g_{1}$ & 6794 & 4181 & 0.00160 & 0.03000 \\
\hline$g_{2}$ & 27128 & 14437 & 0.00080 & 0.01500 \\
\hline$g_{3}$ & 108438 & 55277 & 0.00040 & 0.00750 \\
\hline$g_{4}$ & 433778 & 218269 & 0.00020 & 0.00375 \\
\hline
\end{tabular}

Table 2: Shock-vortex interaction: grid features.

exact solution is not known, the discretization error of a numerical solution and the convergence properties of a numerical scheme can still be estimated using a suite of techniques collectively known as Richardson extrapolation (RE). There exist different variations of the RE technique depending on the number of grid levels involved and on the expression of the leading error terms that is a priori assumed in the analysis; hereafter we refer to the generalized RE technque of fixed order, following the nomenclature used in [19]. The term generalized refers to the fact that the RE technique is applied to $n$ th-order-accurate schemes and grids refined by an arbitrary factor $r$. Note that the use of nested triangulations fixes the grid refinement ratio between each couple of meshes in the sequence to a value $r=2$. It is also assumed that the order of convergence $n$ is known (hence, the term fixed) and is typically set equal to the design order of the scheme. The method used here relies on an error expansion of the type

$$
\varepsilon_{h}(x)=u_{h}(x)-u_{0}(x)=\sum_{p=n}^{\infty} g_{p}(x) h^{p}=g_{n}(x) h^{n}+H O T
$$

Neglecting the HOT, writing Eq. (13) for two grid levels $h$ and $r h$, and solving for the unknown exact solution, one obtains:

$$
\tilde{u}_{0}=u_{h}-\frac{u_{r h}-u_{h}}{\left(r^{n}-1\right)}
$$

In Eq. (14) we have used a tilde to underline the fact that $\tilde{u}_{0}$ is only an approximation of the corresponding quantity that appears in Eq. (13), because the higher-order contributions have been neglected.

Since an exact solution is not known, for the present test-case, Eq. (14) has been used as follows:

$$
\tilde{S}_{0}=S_{h_{4}}-\frac{S_{h_{3}}-S_{h_{4}}}{3}
$$

to provide an approximation of the exact entropy integral of Eq. (12). This is a rather common practice that, according to Roache [17], has been in use in the CFD arena since the early 1990s, even though the procedure is somewhat questionable, because the order is a-priori given in order to perform the RE.

The results obtained with this procedure are summarized in the convergence plots of Fig. 7. The plots show that all the captured solutions converge at best with a first order rate. When removing the error due to the shock-capturing, all the schemes 
behave as if the solution were smooth. In particular, all the schemes provide convergence rates very close to those observed in [16]: 2 for the liner LDA and SU schemes, very close to 2 for the $\mathrm{Bc}$ scheme, a rate of roughly 1.5 for the LDAN scheme, and an order 1 for the $\mathrm{N}$ scheme.

\section{Conclusion and outlook}

We have provided a preliminary study of the behaviour of explicit ALE residual distribution when coupled with shock-fitting. The results show that the fitted solutions are quite independent on the stabilization mechanism used. Multidimensional upwind schemes, and centered schemes with some form of artificial dissipation yield very similar results. What makes most of the difference is the accuracy of the discretization. Even with fitting, first order schemes fail to provide the correct boundary conditions for the discontinuity, producing a visible error in shock position and strength. high order discretizations, both linear and nonlinear, are thus required. A comparison with captured solutions, shows two main results: fitting allows to recover second order of accuracy where capturing leads to first order; the fitted results are much less sensitive to the use of monotonicity preserving methods, as well as to mesh irregularities due to the absence of mesh dependent errors generated in the captured region. In addition anomalies related to the mesh dependent nature of the capturing error error are removed so that the methods behave as if the flow were smooth. This has been proven on two test cases.

Natural extensions of this work will be to consider more complex cases, and more interesting discretization strategies. The first aspect may involve stronger shockvortex interactions, with the appearance of secondary (lambda) shocks. Concerning the discretization, the ALE framework used here would allow quite naturally the use of a hybrid fitting/adaptive approach, with an $r$-adaptation strategy in the nonfitted domains, as proposed e.g. in [3]. Lastly, a very interesting test would be to repeat the studies of this paper when the fitting is performed using non-conservative formulations in the CFD part. This may reveal very useful when considering flows also involving strong contact discontinuities, and chemistry.

Acknowledgements The authors are indebted to R. Paciorri for the many constructive discussions during the development of this work.

\section{References}

1. Abgrall, R., Mezine, M.: Construction of second order accurate monotone and stable residual distribution schemes for unsteady flow problems. Journal of Computational Physics 188(1), 16 - 55 (2003). DOI http://dx.doi.org/10.1016/S0021-9991(03)00084-6. URL http://www.sciencedirect.com/science/article/pii/S0021999103000846 
2. Arina, R., Marsilio, R.: A shock-fitting spectral method for the study of shock-vortex interactions. In: 28th Fluid Dynamics Conference. American Institute of Aeronautics and Astronautics (AIAA) (1997). DOI 10.2514/6.1997-1839

3. Arpaia, L., Ricchiuto, M.: r-adaptation for Shallow Water flows: conservation, well balancedness, efficiency. Research Report RR-8956, Inria Bordeaux Sud-Ouest (2016). URL https://hal.inria.fr/hal-01372496

4. Arpaia, L., Ricchiuto, M., Abgrall, R.: An ale formulation for explicit runge-kutta residual distribution. J. Sci. Comput. 190(34), 1467-1482 (2014)

5. Arpaia, L., Ricchiuto, M., Abgrall, R.: An ALE formulation for explicit rungekutta residual distribution. Journal of Scientific Computing pp. 1-46 (2014). DOI 10.1007/s10915-0149910-5. URL http://dx.doi.org/10.1007/s10915-014-9910-5

6. Bonfiglioli, A., Grottadaurea, M., Paciorri, R., Sabetta, F.: An unstructured, three-dimensional, shock-fitting solver for hypersonic flows. Computers and Fluids 73, 162-174 (2013)

7. Bonfiglioli, A., Paciorri, R., Campoli, L.: Unsteady shock-fitting for unstructured grids. International Journal for Numerical Methods in Fluids 81(4), 245-261 (2016). DOI 10.1002/fld.4183. Fld.4183

8. Grasso, F., Pirozzoli, S.: Shock-wave-vortex interactions: Shock and vortex deformations, and sound production. Theoretical and Computational Fluid Dynamics 13(6), 421-456 (2000). DOI 10.1007/s001620050121. URL http://dx.doi.org/10.1007/s001620050121

9. Hughes, T., Scovazzi, G., Tezduyar, T.: Stabilized methods for compressible flows. J. Sci. Comp. 43, 343-368 (2010)

10. Inoue, O., Hattori, Y.: Sound generation by shock-vortex interactions. Journal of Fluid Mechanics 380, 81 - 116 (1999). DOI doi:10.1017/S0022112098003565

11. Meadows, K.R., Kumar, A., Hussaini, M.: Computational study on the interaction between a vortex and a shock wave. AIAA Journal 29(2), 174-179 (1991). DOI 10.2514/3.59916. URL http://dx.doi.org/10.2514/3.59916

12. Paciorri, R., Bonfiglioli, A.: A shock-fitting technique for $2 \mathrm{D}$ unstructured grids. Computers and Fluids 38(3), 715-726 (2009)

13. Paciorri, R., Bonfiglioli, A.: Shock interaction computations on unstructured, twodimensional grids using a shock-fitting technique. Journal of Computational Physics 230(8), 3155 - 3177 (2011). $\quad$ DOI http://dx.doi.org/10.1016/j.jcp.2011.01.018. URL http://www.sciencedirect.com/science/article/pii/S0021999111000362

14. Pao, S., Salas, M.: A numerical study of two-dimensional shock vortex interaction. In: Fluid Dynamics and Co-located Conferences, pp. - . American Institute of Aeronautics and Astronautics (1981). DOI 10.2514/6.1981-1205. URL http://dx.doi.org/10.2514/6.1981-1205

15. Ricchiuto, M.: An explicit residual based approach for shallow water flows. J.Comput.Phys. 80, 306-344 (2015)

16. Ricchiuto, M., Abgrall, R.: Explicit Runge-Kutta residual distribution schemes for time dependent problems: Second order case. Journal of Computational Physics 229(16), 5653 - 5691 (2010). DOI 10.1016/j.jcp.2010.04.002. URL http://www.sciencedirect.com/science/article/pii/S0021999110001786

17. Roache, P.: Quantification of uncertainty in computational fluid dynamics. ANNUAL REVIEW OF FLUID MECHANICS 29, 123-160 (1997). DOI 10.1146/annurev.fluid.29.1.123

18. Rossiello, G., De Palma, P., Pascazio, G., Napolitano, M.: Second-order-accurate explicit fluctuation splitting schemes for unsteady problems. Computers \& Fluids 38(7), 1384 - 1393 (2009). DOI 10.1016/j.compfluid.2008.01.021. Special Issue Dedicated to Professor Alain Lerat on the Occasion of his 60th Birthday

19. Roy, C.J., McWherter-Payne, M.A., Oberkampf, W.L.: Verification and Validation for Laminar Hypersonic Flowfields Part 1: Verification. AIAA Journal 41(10), 1934-1943 (2003)

20. Salas, M.: A Shock-Fitting Primer, $1^{s t}$ edn. CRC Applied Mathematics \& Nonlinear Science. Chapman \& Hall (2009)

21. Salas, M.D., Zang, T.A., Hussaini, M.Y.: Shock-fitted Euler solutions to shock-vortex interactions, pp. 461-467. Springer Berlin Heidelberg, Berlin, Heidelberg (1982). DOI 10.1007/3540-11948-5_59 
22. Shewchuk, J.R.: Triangle: Engineering a 2D Quality Mesh Generator and Delaunay Triangulator. In: M.C. Lin, D. Manocha (eds.) Applied Computational Geometry: Towards Geometric Engineering, Lecture Notes in Computer Science, vol. 1148, pp. 203-222. Springer-Verlag (1996). From the First ACM Workshop on Applied Computational Geometry

23. Shu, C.W.: Essentially non-oscillatory and weighted essentially non-oscillatory schemes for hyperbolic conservation laws. In: Advanced numerical approximation of nonlinear hyperbolic equations, pp. 325-432. Springer Berlin Heidelberg (1998) 


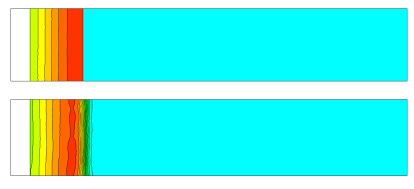

(a) $t=0.4 \mathrm{~N}$ scheme

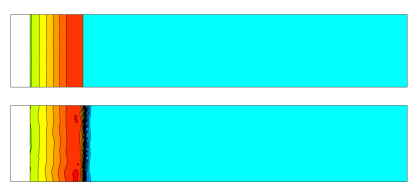

(d) $t=0.4$ LDA scheme

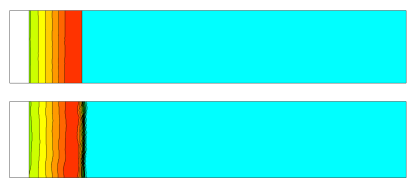

(g) $t=0.4$ LDAN scheme

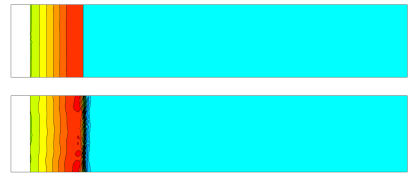

(j) $t=0.4 \mathrm{SU}$ scheme

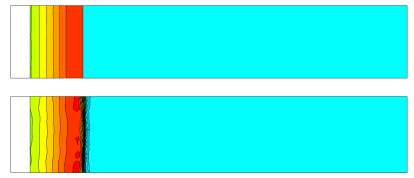

(m) $t=0.4 \mathrm{Bc}$ scheme

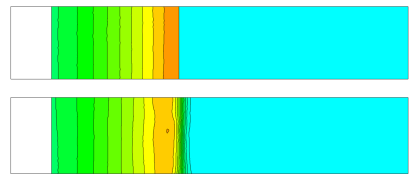

(b) $t=1.2 \mathrm{~N}$ scheme

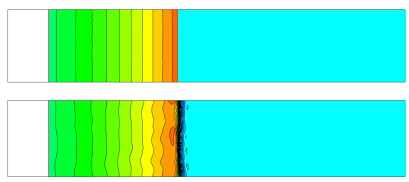

(e) $t=1.2 \mathrm{LDA}$ scheme

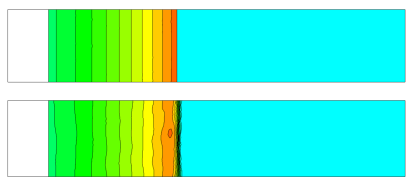

(h) $t=1.2$ LDAN scheme

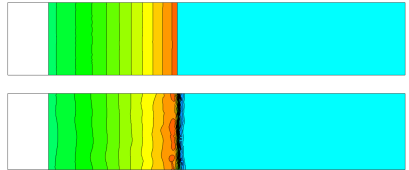

(k) $t=1.2 \mathrm{SU}$ scheme

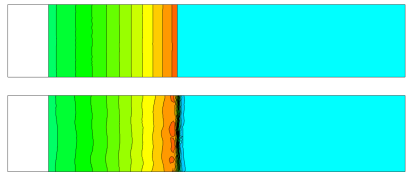

(n) $t=1.2 \mathrm{Bc}$ scheme

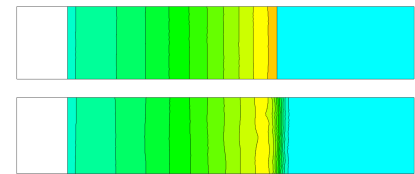

(c) $t=2.0 \mathrm{~N}$ scheme

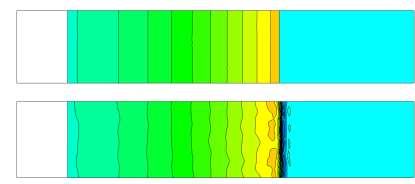

(f) $t=2.0$ LDA scheme

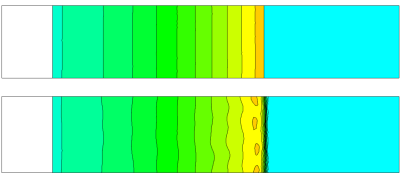

(i) $t=2.0$ LDAN scheme

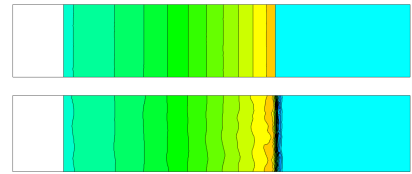

(1) $t=2.0 \mathrm{SU}$ scheme

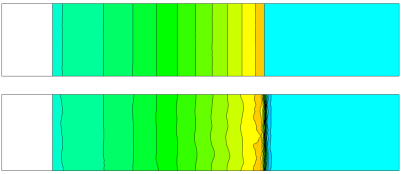

(o) $t=2.0 \mathrm{Bc}$ scheme

Fig. 3: Shock-expansion interaction: pressure contours at times $t=0.4$ (left), $t=1.2$ (centre) and $t=2.0$ (right): 20 equally spaced contour levels between 0.5 and 2.6, fitted solutions on top and captured solutions on the bottom of each frame. 

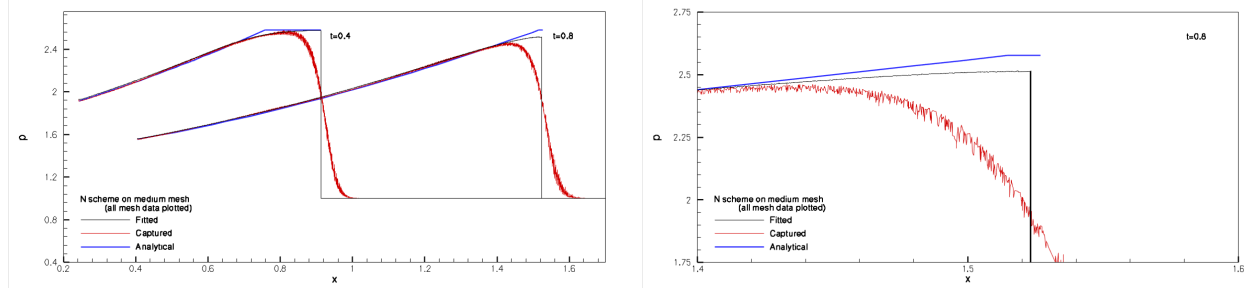

(a) $\mathrm{N}$ scheme
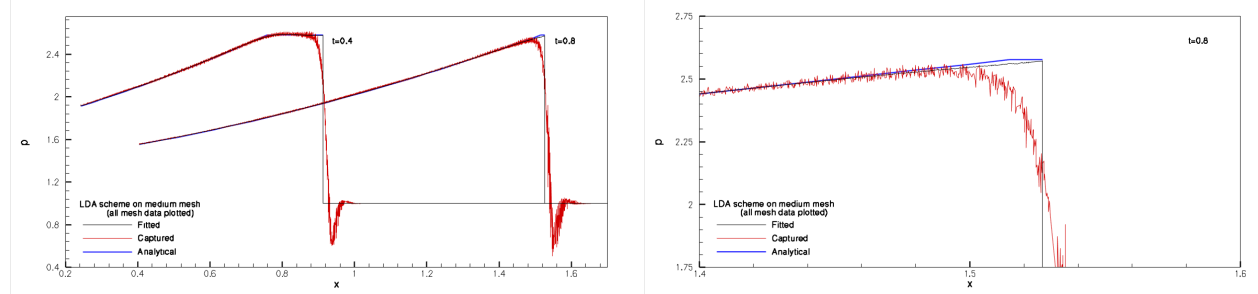

(b) LDA scheme
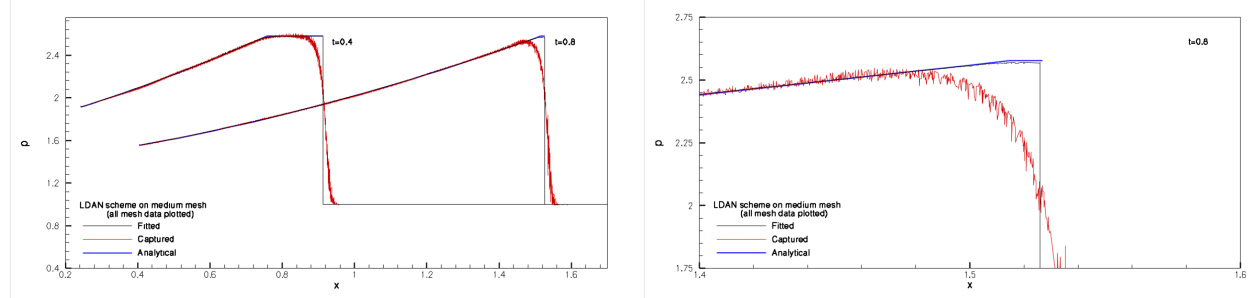

(c) LDAN scheme
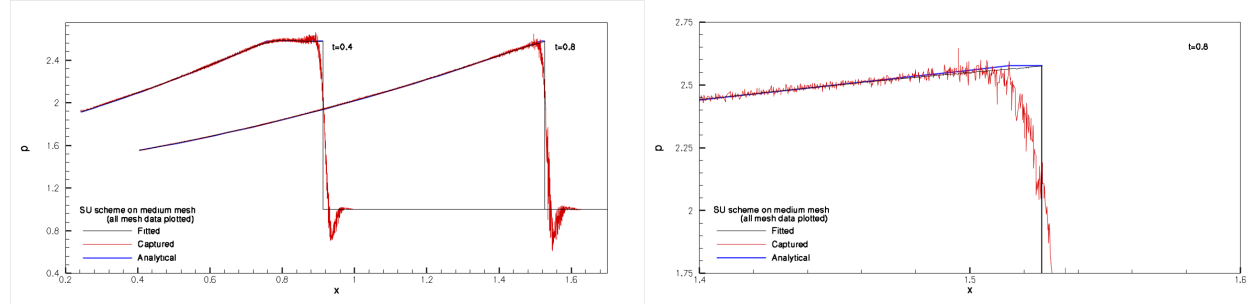

(d) SU scheme
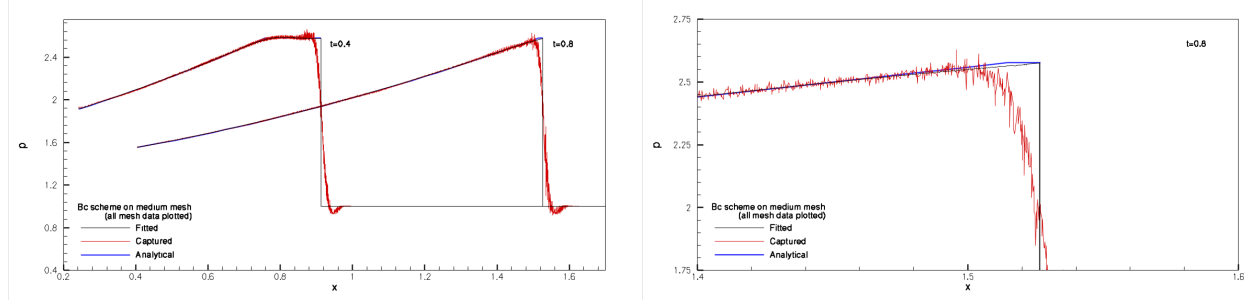

(e) Bc scheme

Fig. 4: Shock-expansion interaction: streamwise pressure profiles at two time instants for all the five spatial discretization schemes. Right column: zoom of the $t=0.8$ peak. 


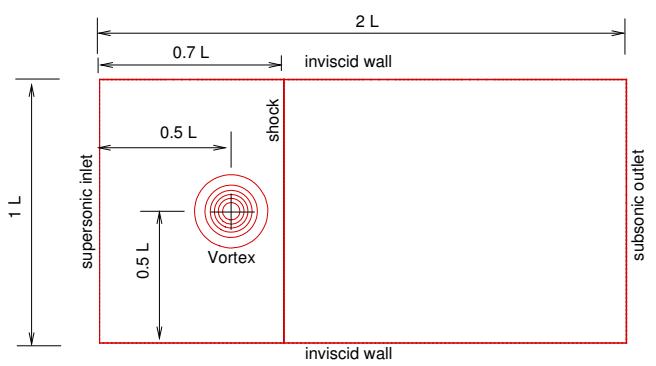

Fig. 5: Shock-vortex interaction: computational domain and boundary conditions.

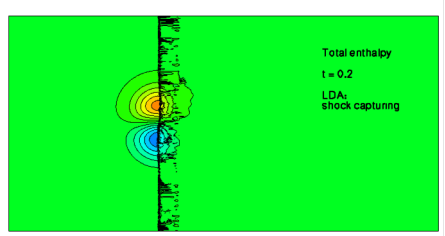

(a) $t=0.2$ LDA (capturing)

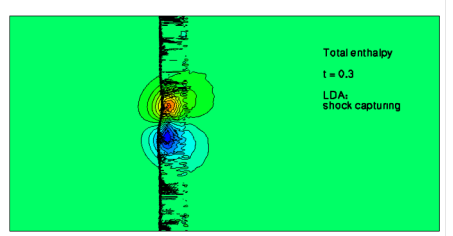

(b) $t=0.3$ LDA (capturing)

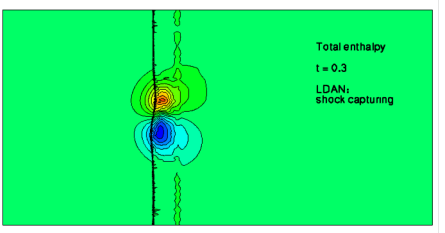

(e) $t=0.3$ LDAN (capturing)

(h) $t=0.3$ LDA (fitting)

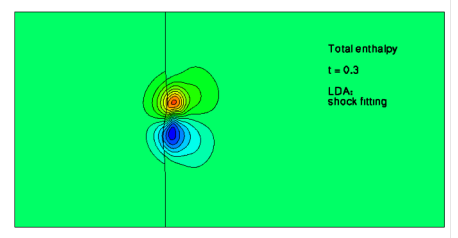

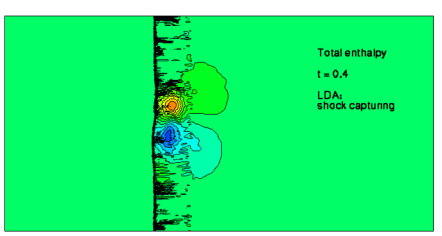

(c) $t=0.4$ LDA (capturing)

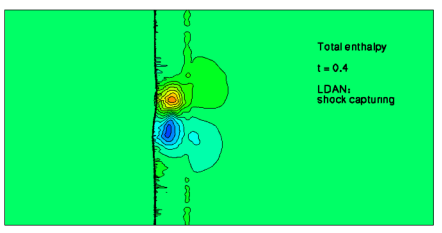

(f) $t=0.4 \mathrm{LDAN}$ (capturing)

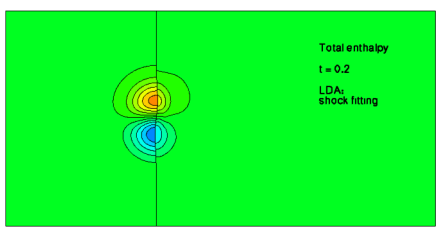

(g) $t=0.2$ LDA (fitting)

Fig. 6: Shock-vortex interaction: total enthalpy contours at times $t=0.2$ (left), $t=0.3$ (centre) and $t=0.4$ (right): 15 equally spaced contour levels obtained with the shock-capturing versions of the LDA (top), and LDAN (middle), and with shockfitting and LDA (bottom). 


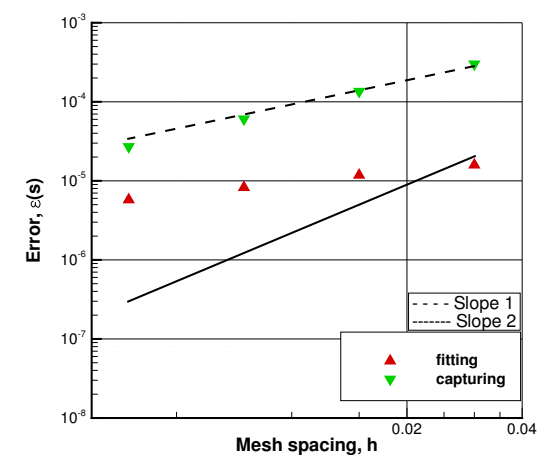

(a) $t=0.4 \mathrm{~N}$ scheme

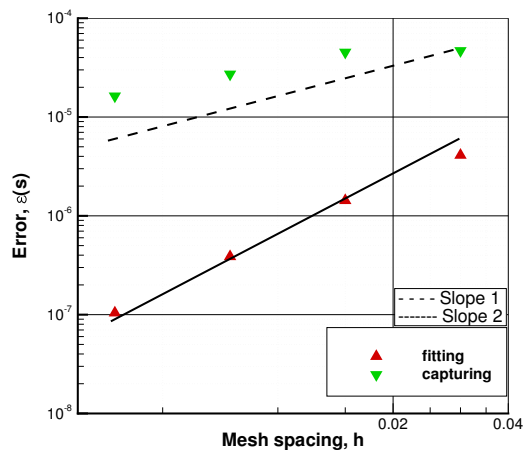

(b) $t=0.4$ LDA scheme

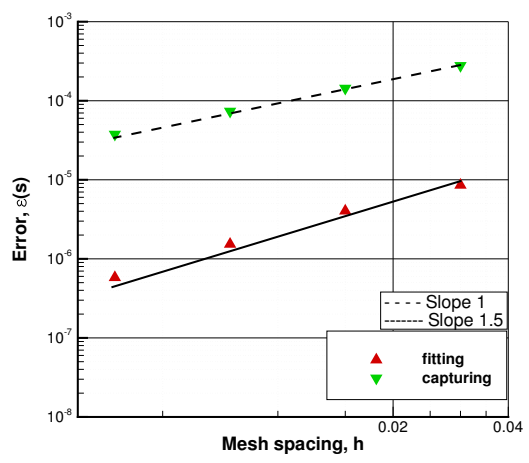

(d) $t=0.4$ LDAN scheme

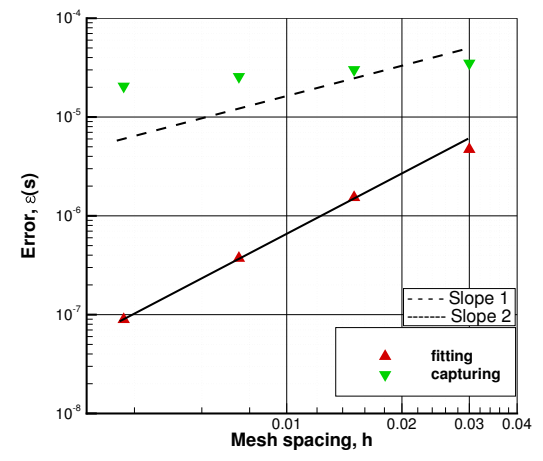

(c) $t=0.4 \mathrm{SU}$ scheme

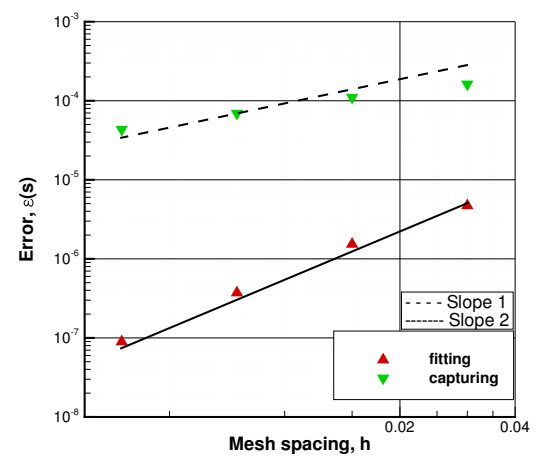

(e) $t=0.4 \mathrm{Bc}$ scheme

Fig. 7: Convergence histories obtained by means of Richardson Extrapolation for the shock-vortex interaction problem at time $t=0.4$. 